\title{
Summary and Guidelines
}

\author{
S.G.O. Johansson ${ }^{\mathrm{a}}$, Tari Haahtela ${ }^{\mathrm{b}}$ \\ aDepartment of Clinical Immunology, Karolinska Hospital, \\ Stockholm, Sweden; 'Skin and Allergy Hospital, \\ Helsinki University Central Hospital, Helsinki, Finland
}

This chapter summarises the key messages of the preceding chapters to provide an overview of the current knowledge base on the prevention of allergy and allergic asthma. Our present knowledge suggests that good progress has been made in our understanding of the underlying principles of prevention of allergy and allergic asthma, and that some prevention strategies can be effective in preventing symptoms or reducing their severity. Major questions remain regarding the prevention of IgE sensitisation, and whether exposure to allergens can be protective or pathogenic, depending upon the time course of allergen exposure in relation to immunological maturity. Much work remains to be done before further firm recommendations can be made.

Recommendations are made for research strategies which may help progress the important areas of primary, secondary and tertiary prevention and thus improve patient care.

\section{Genetics}

Current Knowledge

Individuals with a family history of atopy have an increased risk of developing IgE sensitisation.

Atopic constitution represents a high risk for the development of allergic diseases such as allergic asthma, allergic rhinoconjunctivitis and atopic eczema.

The contribution of genetic factors to the development of $\operatorname{IgE}$ sensitisation and to the family history of an IgE-mediated disease is between $70-80 \%$. The risk of developing asthma, rhinitis or eczema in general is related to a family history of the respective disease. 


\section{Further Actions Required}

Identification of genetic variants that predispose to allergic disease may result in several outcomes.

Early identification of susceptible individuals would allow them to be targeted at early age to prevent IgE sensitisation, and for avoidance of allergen exposure. Genetic screening in early life may become useful in preventing allergic disease.

Identification of environmental factors that interact with susceptibility genes to trigger allergic disease and the periods of life in which this occurs may give opportunities to environmental modification.

Understanding susceptibility factors will help to find new drugs to treat and prevent allergic diseases. Genetic variants influence response to therapy, and their identification will improve the efficacy of therapeutic measures.

\section{Environmental Influences}

\section{Current Knowledge}

Cigarette smoking in pregnancy deteriorates fetal lung development and increases the risk of wheezing in infancy.

Environmental tobacco smoke causes wheeze in infants and aggravates asthma. All children benefit through avoidance of environmental tobacco smoke.

Avoidance of allergen exposure has only been partially successful in the prevention of IgE sensitisation. Avoidance is difficult to implement, benefits are small and long-term effects doubtful.

Infants at high allergy risk (allergic disease in atopic parents or siblings) may benefit through avoidance of pets and dust mites during the 1st year of life.

Some early respiratory infections, e.g. pertussis and bronchiolitis caused by respiratory syncytial virus, as well as some forms of gastro-enteritis may enhance the development of allergic diseases and asthma.

Relative lack of early microbial exposure particularly early in life, may enhance the development of allergic diseases, but further research is needed in this field.

\section{Further Actions Required}

Study priorities in allergy-related environmental issues are:

Exploration of the environmental factors associated with residence in locations with high versus low prevalence of allergy.

Effect of modulating the diet of pregnant and breast-feeding mothers including increased intake of vegetables, fruit, cereals, nuts, starches and fish.

Effect of allergen avoidance in primary and secondary prevention, and in the treatment of allergic diseases (tertiary prevention). 
Effect of indoor air as well as ambient air pollutants (e.g. NOx, ozone, fine particulates) in the development of allergic diseases and their aggravation.

\section{Early Immunological Influences}

\section{Current Knowledge}

Allergen-specific T-helper lymphocyte programming begins early in life and consolidates into the adult pattern during pre-school years. This programming is directly driven by dietary and inhalant allergens that are ubiquitous in the environment.

Early exposure to high levels of dietary or inhalant allergens usually results in high zone tolerance, which protects against IgE sensitisation. Postnatal development of allergen-specific responses is modulated by Th1 function, which develops through the contact with microbial signals being absent from the fetal environment.

Early exposure to low levels of inhalant allergens in the absence of sufficient microbial exposure may trigger low zone tolerance resulting in either weak Th1-like immunity, or Th2-polarised responses. This may increase the risk for $\operatorname{IgE}$ sensitisation.

\section{Further Actions Required}

Study priorities are:

Safe interventions strategies for infants at high allergy risk. As allergic manifestations are also increasingly common in children without family history of allergy, there is a need for 'easy to implement guidelines' for all infants. Allergic diseases are difficult to modify once established.

Role of nutritional factors in infants' immune function and lung development.

Possibilities to induce postnatal high zone tolerance by administering high doses of allergens.

Inhibition of Th2 function during the early stages of allergy development by new antagonists (against IgE, IL-4 and IL-12 for example) alone or in combination with allergen-specific immunotherapy.

Effect of anti-viral agents to treat bronchiolitis induced by respiratory syncytial virus and to prevent asthma in young children at high risk.

\section{Predictive and Early Diagnosis}

\section{Current Knowledge}

Family history, taken by an experienced clinician, is the most reliable predictor of development of allergy and asthma in infants. If both parents 
have allergic asthma, allergic rhinoconjunctivitis, or atopic eczema, the child has a fourfold risk to develop allergies compared with a child whose parents do not have allergies. If only one parent has allergic disease, the risk is twofold.

Early signs of an allergic disease, especially atopic eczema, and presence of IgE antibodies to inhalant allergens are important risk factors for later respiratory allergy. IgE antibodies in infant serum to basic food proteins, e.g. hen's egg, may predict the so-called allergic march.

Current immunological markers obtained during the neonatal period are not specific or sensitive enough to predict allergic disease. High IgE concentration in cord blood or infant serum is specific for subsequent allergic disease but has a low sensitivity, and measuring total $\mathrm{IgE}$ is not recommended for screening.

\section{Further Actions Required}

Mechanisms of initial IgE sensitisation should be explored to develop markers necessary for early prediction.

\section{Preventive measures}

\section{Guidelines}

Primary Prevention

Avoid smoking and exposure to environmental tobacco smoke, particularly during pregnancy and early childhood (B). Tobacco smoke should be also removed from work places (B).

Avoid damp housing conditions (C), and reduce indoor air pollutants (C).

Breast-feed exclusively until 4-6 months $\left(\mathrm{B}^{1}\right)$. No special diet for the lactating mother (A).

Reduce exposure to inhalant allergens in young children at high risk (house dust mites, cockroaches, furred pets) (B).

Eliminate sensitizing and highly irritating agents in occupational environments $(\mathrm{C})$. If this is not possible, implement measures to prevent employee exposure.

${ }^{1}$ Based on allergy prevention studies, exclusive breast-feeding and avoidance of solid foods at least 4 months seems to be effective. WHO dietary guidelines recommend exclusive breast-feeding for 6 months in general. Infants who are not breast-fed because their mothers are HIV positive, and who have consequently developed allergy to cow's milk, should receive hypoallergenic formula, if available, to improve symptom control (B). 


\section{Secondary Prevention}

Treat atopic eczema in infants and children to try to prevent respiratory allergy (D).

Treat upper airway disease (rhinoconjunctivitis) to reduce the risk of development of asthma (D).

In young children already sensitised to house dust mites, pets or cockroaches, exposure should be reduced to prevent onset of allergic disease (B).

Remove employees from occupational exposure, if they have developed symptoms caused by occupational allergic sensitisation (C).

\section{Tertiary Prevention}

Infants with cow milk allergy should avoid cow milk proteins; if a supplement is needed, use hypoallergenic formula, if available/affordable, to improve symptom control (B).

Patients with asthma, rhinoconjunctivitis or eczema who are allergic to indoor allergens such as house dust mites, cockroaches and animal danders should eliminate or reduce the exposure to improve symptom control and prevent exacerbations $(\mathrm{A}-\mathrm{B})$.

Aim pharmacotherapy primarily towards the underlying inflammatory process (A).

Avoid strictly acetylsalicylic acid or other non-steroidal anti-inflammatory drugs in patients who have been diagnosed to be sensitive to them (C).

\section{Further Actions Required}

Study priorities are:

Detection of potent allergens in marketed ready-made foods (also hidden allergens). Declaration of the food allergen content should be improved.

Effect of human milk, and the role of milk constituents (e.g. n3/n6 fatty acids, $\alpha$-linolenic acid and cytokines) in the development of sensitisation and clinical manifestations of allergy.

Effect of dietary factors in the development of allergy and asthma, e.g. salt intake, antioxidants and $\mathrm{n} 3 / \mathrm{n} 6$ fatty acids.

Effect of intestinal microbial flora, antibiotics, pro- and pre-biotics, and microbial vaccinations in the development of sensitisation and clinical manifestations of allergy.

Effect of low-allergen domestic environments in sensitised children and in patients with allergic diseases. Environmental measures should be studied in terms of clinical benefit, feasibility, compliance, costs, and the interventions should be of adequate length, e.g. 12 months. 
Effect of house dust mite avoidance on allergic symptoms, exacerbation rates, need for medication and health costs.

Role of indoor air and ambient air pollutants in the development of sensitisation and clinical manifestations of allergy.

Further Actions for Occupational Allergies

Risks of occupational allergy should be monitored and epidemiological information collected by a globally agreed questionnaire.

High risk for allergy environments should be identified (e.g. enzymes, flours, latex and animals). In atopic employees who work in these environments, detection of sensitisation by skin prick tests or IgE antibody measurements could prevent the development of clinical allergy.

General principles of prevention of occupational asthma, rhinoconjunctivitis and dermatitis (e.g. hand eczema or contact dermatitis) should be published by national regulatory and advisory bodies.

\section{Education}

Guidelines

Patient education regarding precipitants of allergic symptoms, asthma, and anaphylaxis is essential. Guided self-management to prevent, assess and treat symptoms is the key to optimising disease control (A).

School policies on asthma and anaphylaxis management are essential (D).

\section{Further Actions Required}

Initiation of an International Coalition for Allergy and Asthma Prevention. The aims are:

To collect effective programs into a database for all countries.

To establish an annual convention on allergy and asthma prevention, education and applied research for medical professionals from participating countries.

To establish an International Allergy and Asthma Prevention \& Education Promotion Fund, supported by public and private funding.

To determine criteria to distribute funds as:

- to seed money to initiate programme activities, and

- to match funds to supplement the available funding.

To determine the criteria for membership of the International Coalition as an Allergy and Asthma Preventing \& Education Promoting Country. 


\section{Costs of Allergies and Asthma}

\section{Guideline}

When considering any intervention for prevention or treatment, take into account not only the evidence showing the beneficial effect of a certain product or measure but also the costs. Use the most cost-effective product or measure.

\section{Further Actions Required}

Study priorities are:

Health economical impact of asthma, rhinitis and eczema and the conditions together, especially in terms of indirect costs.

Societal costs of allergy and asthma in developing countries, and the development of these costs.

Health economic evaluations in all controlled studies exploring early treatment of allergic disease, especially asthma.

Health economic impact of measures taken to control environmental exposure compared with medication management in prevention and treatment of allergies and asthma.

Development of more advanced models to study the economic impact of allergic diseases on health.

Prof. S.G.O. Johansson, MD, PhD

Department of Clinical Immunology

Karolinska University Hospital

SE-171 76 Stockholm (Sweden) 